\title{
Transatlantica
}

Revue d'études américaines. American Studies Journal

\section{Atelier-conférence : Smaro Kamboureli « From CanLit to Canlits : The Re-formation of a Discipline »}

Université Sorbonne Nouvelle - Paris 3, 17 novembre 2015

\section{Samuel Harvet}

\section{Q OpenEdition}

Journals

Édition électronique

URL : https://journals.openedition.org/transatlantica/7673

DOI : $10.4000 /$ transatlantica.7673

ISSN : $1765-2766$

Éditeur

Association française d'Etudes Américaines (AFEA)

Référence électronique

Samuel Harvet, «Atelier-conférence : Smaro Kamboureli « From CanLit to Canlits : The Re-formation of a Discipline » », Transatlantica [En ligne], 2 | 2015, mis en ligne le 31 mai 2016, consulté le 10 février 2023. URL : http://journals.openedition.org/transatlantica/7673 ; DOI : https://doi.org/10.4000/ transatlantica.7673

Ce document a été généré automatiquement le 10 février 2023.

Creative Commons - Attribution - Pas d'Utilisation Commerciale - Pas de Modification 4.0 International - CC BY-NC-ND 4.0

https://creativecommons.org/licenses/by-nc-nd/4.0/ 


\title{
Atelier-conférence : Smaro Kamboureli « From CanLit to Canlits : The Re-formation of a Discipline »
}

Université Sorbonne Nouvelle - Paris 3, 17 novembre 2015

\author{
Samuel Harvet
}

1 Cette première séance de l'année du séminaire "Les discours de la mondialisation ", organisé par Christine Lorre-Johnston à l'université Sorbonne Nouvelle-Paris 3, a accueilli la professeure Smaro Kamboureli, Avie Bennett Chair in Canadian Literature à l'université de Toronto. Smaro Kamboureli est une spécialiste de littérature canadienne et de théorie critique contemporaine. Elle a rendu compte des recherches qu'elle mène depuis une quinzaine d'années sur l'épistémologie de la littérature canadienne contemporaine. Ces recherches s'inscrivent dans le cadre du TransCanada project, qui a donné lieu à trois grands colloques depuis son lancement, et à la publication de deux volumes de contributions (Kamboureli et Zacharias, 2012; Kamboureli et Verduyn, 2014). Le $4^{\text {ème }}$ colloque aura lieu à Toronto en 2017, à l'occasion des 150 ans de l'État canadien, avec notamment pour invitée l'écrivaine Larissa Lai.

\section{Déconstruire « la littérature canadienne »}

Les recherches de Smaro Kamboureli s'insèrent dans la perspective d'une approche transnationale de la littérature canadienne, qui entend dépasser le modèle théorique du multiculturalisme, dont on connaît au Canada l'influence théorique, sociale et politique. Il s'agit pour Kamboureli d'envisager une voie féconde pour dépasser ce modèle, qui a constitué un précieux paradigme pour penser l'intégration pacifiée des composantes très diverses de la société, mais qui s'est transformé, en s'institutionnalisant, en un quasi obstacle épistémologique pour renouveler l'appréhension des diverses tendances de la littérature canadienne, tout 
particulièrement des écritures migrantes et autochtones. La redécouverte de ces écritures implique ainsi une déconstruction de la littérature canadienne comme discours disciplinaire (Foucault, 1970), des partitions entre le dedans et le dehors de la discipline, et de la manière dont elle a pu être instrumentalisée par l'État-nation comme symbole d'une société unifiée. Ce geste critique ouvre deux perspectives, socioculturelle et épistémologique : c'est, d'une part, la définition même de l'État-nation canadien qui est mise en cause, notamment à travers la réinsertion des littératures autochtones au sein de "CanLits» pluralisées. D'autre part, une telle démarche interroge le rôle du chercheur littéraire et la part d'engagement de son travail, qui vise notamment à interroger l'inconscient politique du champ littéraire canadien tel qu'il s'est constitué (Kamboureli, 2012, p. 17). À l'horizon, il s'agit de donner à lire la littérature canadienne comme une dynamique en plein essor, plutôt que comme un corpus homogène bien balisé.

\section{Faire face aux évolutions des humanités}

3 Le champ de recherches ouvert par le TransCanada project s'insère en effet dans le tournant éthique amorcé dans les cultural studies nord-américaines depuis la fin des années 1980, à partir notamment des travaux de Paul Ricœur, Martha Nussbaum et Richard Rorty (Garber, Hanssen et Walkowitz, 2000). Ce tournant peut être interprété comme une réponse possible des humanités à la mise en question de leur valeur sociale. En rupture avec l'éthique de la lecture prônée par un poststructuraliste tel que J. Hillis Miller, Smaro Kamboureli insiste toutefois sur le caractère plus global que doit adopter une telle approche, en amont comme en aval du moment herméneutique proprement dit, à travers notamment le choix du sujet, du style pour en parler, ou bien des modes de diffusion des résultats de recherche. Elle rejette ainsi une vision dominante d'après elle, dans la pensée néolibérale, selon laquelle le rôle de l'université se limite à être un forum d'expression des différences et des discriminations, ce qui tend à désamorcer son potentiel de déstabilisation de l'ordre social.

4 Le TransCanada project interroge la notion de collaboration au sein des sciences humaines qui ne peut, selon Kamboureli, se contenter d'importer un modèle méthodologique hérité des sciences sociales, mais nécessite une refonte de ses méthodes et de ses critères d'évaluation. Dans le sillage d'une série d'essais qu'elle a consacrés à ces questions, Kamboureli souligne les risques cachés qu'a entraînés la promotion de l'interdisciplinarité, en tendant à privilégier une vision quantifiable et appliquée du savoir, inappropriée au champ des études littéraires (Kamboureli et Coleman, 2011).

\section{" Different, but the same »}

Smaro Kamboureli présente ensuite l'actualité du TransCanada project, centrée sur la notion de parenté (kinship), comprise non dans son sens généalogique, mais comme un trope qui permet de relier les voix autochtones et celles qui sont mieux représentées de la littérature canadienne. Travailler sur une telle notion soulève des enjeux à la fois méthodologiques et politiques: il s'agit de trouver un moyen de dialoguer avec les cultures autochtones sans les folkloriser ni essentialiser les différences interculturelles. La notion de parenté ainsi redéfinie peut fournir un outil conceptuel puissant pour 
identifier, hors du prisme du "Même » et de l' "Autre ", les points de contact entre communautés culturelles, d'où peuvent surgir des espaces de parole. C'est avant tout un instrument à vocation heuristique : il ne s'agit pas véritablement d'œuvrer à un rapprochement des communautés. Kamboureli rapproche cette démarche de celle de la Commission Vérité et Réconciliation, dont la composition était mixte entre Canadiens d'origine autochtone et immigrée.

Smaro Kamboureli a conclu sa communication en présentant sa récente collaboration avec l'écrivaine autochtone, membre de la nation stó :lō, Lee Maracle, dont elle a édité les discours (oratories) dans le livre Memory Serves (Maracle, 2015). Ce recueil de traces orales translittérées par Lee Maracle porte les marques d'une certaine hybridation de la langue anglaise par le rythme et le lexique hérités des langues autochtones. La conférencière note que Lee Maracle est moins l'auteure de ses contes que leur interprète : elle les a traduits à sa manière, fidèle à l'esprit plutôt qu'à la lettre d'un corpus oral non stabilisé. Maracle a recontextualisé ces histoires dans une adaptation créative, en accomplissant un véritable travail de remédiation qui pallie les chaînons manquants de la culture orale autochtone. Fruit d'une collaboration de plus de sept ans entre l'auteure et son éditrice, ce travail incarne l'engagement éthique du chercheur qui met en jeu sa responsabilité dans la transmission d'une parole minorée.

Une première question de l'assistance a porté sur le lien que tisse l'individu avec sa parenté : peut-on définir une personne canadienne comme autochtone ou non, en dehors de critères phylogénétiques ? Peut-on choisir sa parenté ? Smaro Kamboureli a répondu en rappelant que si les liens de sang ne sont généralement pas pris en compte au Canada pour définir la condition d'autochtone (à l'exception de la tribu des Mohawk après la crise d'Oka), les anthropologues montrent bien comment l'appartenance ethnique reste un critère d'affiliation identitaire de premier ordre dans tout groupe social.

Pour plus d'informations sur le séminaire, vous pouvez consulter la page suivante: http://www.thalim.cnrs.fr/seminaires-et-formations/article/les-discours-de-laglobalisation?id_evenement=2483\&lang=fr\#evenement_2483

\section{BIBLIOGRAPHIE}

FOUCAULT, Michel, L'ordre du discours, Paris, Seuil, 1970.

GARBER, Marjorie, Beatrice HANSSEN et WALKOWITZ Rebecca (dir.), The Turn to Ethics, Londres, Routledge, 2000.

KAMBOURELI, Smaro et Robert ZACHARIAS (dir.), Shifting the Ground of Canadian Literary Studies, Waterloo, Wilfrid Laurier University Press, 2012.

KAMBOURELI, Smaro et Christl VERDUYN (dir.), Critical Collaborations: Indigeneity, Diaspora, and Ecology in Canadian Literary Studies, Waterloo, Wilfrid Laurier University Press, 2014. 
KAMBOURELI, Smaro et Daniel COLEMAN (dir.), Retooling the Humanities: The Culture of Research in Canadian Universities, Edmonton, University of Alberta Press, 2011.

MARACLE, Lee, Memory Serves, textes réunis par Smaro Kamboureli, Edmonton, NeWest Press, 2015.

\section{AUTEUR}

\section{SAMUEL HARVET}

THALIM, Université Sorbonne Nouvelle - Paris 3 